\title{
Functional Complex Point-Defect Structure in a Huge-Size-Mismatch System
}

\author{
R Ishikawa ${ }^{1}, \mathrm{~N} \mathrm{Shibata}^{2}, \mathrm{~F} \mathrm{Oba}^{3}$, T Taniguchi ${ }^{4}, \mathrm{~S}$ D Findlay ${ }^{5}$, I Tanaka ${ }^{3,6}$ and Y Ikuhara ${ }^{2,6}$ \\ 1. Materials Science and Technology Division, Oak Ridge National Laboratory, Tennessee, USA. \\ 2. Institute of Engineering Innovation, University of Tokyo, Tokyo, Japan. \\ 3. Department of Materials Science \& Engineering, Kyoto University, Kyoto, Japan. \\ 4. Advanced Key Technologies Division, National Institute for Materials Science, Tsukuba, Japan. \\ 5. School of Physics, Monash University, Victoria Australia. \\ 6. Nanostructures Research Laboratory, Japan Fine Ceramics Center, Nagoya, Japan.
}

Cubic boron nitride $(c-\mathrm{BN})$ is a promising host material in various optoelectronic applications such as photoemitters, scintillators and light-emitting diodes in the UV or visible range. Owing to its excellent hardness (next to diamond) and strong covalent bonding nature, it has been extremely difficult to effectively dope functional impurities. Recently, crystal growth by using reactive flux with the temperature gradient method at high pressure and high temperature $(5.5 \mathrm{GPa}, 1773 \mathrm{~K})$ has achieved stable doping of luminous rare-earth (RE) atoms into $c$-BN host lattice, and we have firstly produced $c$ $\mathrm{BN}: \mathrm{RE}$ single crystals with visible light luminescence (blue-colored luminescence in Fig. 1(a)) ${ }^{1.2}$. To understand the doping mechanism and emergence of luminous properties, the fundamental question remains as to how RE dopants with a huge size mismatch can be accommodated within high-density $c$ $\mathrm{BN}$ crystal lattices. Here we show the direct determination of the exact atomic sites and valence states of individual Ce dopants buried inside $c$-BN single crystals using atomic-resolution scanning transmission electron microscopy (STEM) and electron energy-loss spectroscopy (EELS) ${ }^{2}$.

Figure 1(b) shows a typical annular dark-field (ADF) STEM image of $c$-BN:Ce viewed along the [110] direction. Randomly distributed bright spots highlight the Ce containing atomic columns, which clearly reveals that the $\mathrm{Ce}$ atoms are not form Ce-clusters but are distributed in the $c$-BN lattices as single atoms. In the higher magnified image (Fig. 1(c), (d)), the sub-angstrom BN dumbbells $(0.9 \AA)$ are clearly resolved, and moreover it is found that all the cationic Ce dopants substitute for the exact $\mathrm{N}$ antisite $\left(\mathrm{Ce}_{\mathrm{N}}\right)$, not for the cationic $\mathrm{B}$ site (Fig. (e)). The valence state of those single Ce atoms was investigated using column-by-column EELS (Fig. 1(f)). By comparison to the EELS spectra of $\mathrm{Ce}^{3+}$ in $\mathrm{Ce}_{2} \mathrm{O}_{3}$ and $\mathrm{Ce}^{4+}$ in $\mathrm{CeO}_{2}$, the valence state of the $\mathrm{Ce}$ atoms is determined to be $3+$, which is doubly checked by $M_{4} / M_{5}$ ratio with second derivative spectrum.

To determine the stable $\mathrm{Ce}$ atom configurations in $c$-BN lattice, we consider all the possible defect structures and evaluated their relaxed structures, charge states and the formation energies using hybrid Hartree-Fock density functional calculations. Consequently, we found that the Ce dopants are incorporated by introduing the complex point defect structure of $\left(\mathrm{Ce}_{N}-4 \mathrm{~V}_{\mathrm{B}}\right)^{6-}$ : Ce atom is located at antisite, surrounded with four B vacancies and the defect is negatively charged. Our findings indicate that even extremely large size mismatch can be stably doped into host lattice by means of the formation of complex point defect structures.

\section{References}

[1] A Nakayama et al, Appl. Phys. Lett., 87 (2005) 21191.

[2] R Ishikawa et al, Phys. Rev. Lett., 110 (2013) 065504. 
[3] This research was supported by a Grand-in-Aid for Scientific Research on Priority Areas, "AtomicScale Modification" from MEXT of Japan and FIRST program. A part of this research was conducted in "Research Hub for Advanced Nano Characterization, The University of Tokyo", supported under "Nanotechnology Platform”. Dr R Ishikawa acknowledges support from JSPS Postdoctoral Fellowships for Research Abroad. Dr N Shibata and Dr F Oba acknowledge support from JSPS KAKENHI, Grand No. 23686093, 23686089, respectively. Dr S D Findlay acknowledges support by the Australian Research Council.

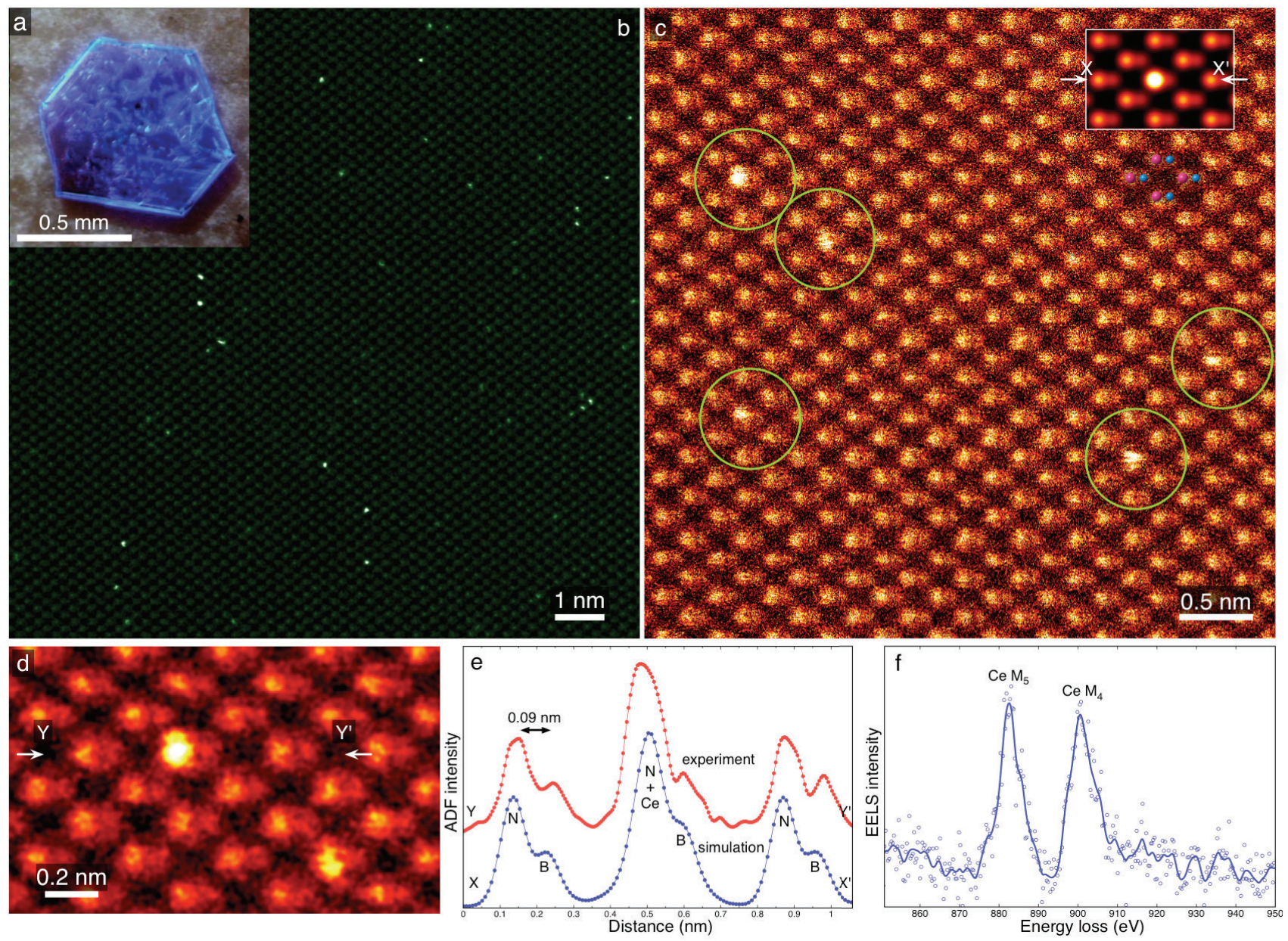

Figure 1. (a) Cathodolumeniscence optical image of $c-\mathrm{BN}$ :Ce single crystal. (b) Typical and (c) atomicresolution ADF STEM image of $c-\mathrm{BN}$ :Ce viewed along the [110] direction and simulated image (inset). (d) Enlarged ADF STEM image with Ce dopants. (e) Intensity profiles obtained along X-X' and Y-Y'. (f) Core-loss spectrum obtained from a single nitrogen atom column containing one Ce atom. 